\title{
Níveis mais Baixos de Fetuína-A Sérica estão Associados a um Maior Risco de Mortalidade em Dez Anos em Pacientes com Infarto do Miocárdio por Supradesnivelamento do Segmento ST
}

\author{
Lower Serum Fetuin-A Levels are Associated with a Higher Ten-Year Mortality Risk in Patients with ST-Elevation \\ Myocardial Infarction
}

Hakan Çakır, ${ }^{10}$ Selçuk Kanat, ${ }^{2}$ Hilal Çakır, ${ }^{3}$ Erhan Tenekecioğlu ${ }^{2}$

Kartal Kosuyolu Training and Research Hospital, ${ }^{1}$ Istanbul - Turquia

Bursa Yuksek Ihtisas Training and Research Hospital, ${ }^{2}$ Bursa - Turquia

Pendik State Hospital, ${ }^{3}$ Istanbul - Turquia

\section{Resumo}

Fundamento: A fetuína-A é um fator anti-inflamatório e anticalcificação envolvido no curso da doença arterial coronariana (DAC). Em alinhamento com essas funções, investigou-se a fetuína-A como marcador de risco cardiovascular em vários estudos. Porém, a associação entre a fetuína-A e o prognóstico dos pacientes com DAC ainda é controversa.

Objetivos: O presente estudo foi conduzido para identificar a associação entre o nível de fetuína-A sérica e doença cardiovascular (DCV) de longo prazo e a mortalidade global por infarto do agudo do miocárdio por supradesnivelamento do segmento ST (STEMI).

Métodos: Foram cadastrados no estudo cento e oitenta pacientes consecutivos com STEMI. A população do estudo foi dividida em subgrupos (mais baixo, $\leq 288 \mu \mathrm{g} / \mathrm{ml}$; e mais alto, $>288 \mu \mathrm{g} / \mathrm{ml}$ ) de acordo com a mediana do nível de fetuína-A. Dados de acompanhamento clínico foram obtidos por contato telefônico anual com pacientes ou familiares. As causas das mortes também foram confirmadas pelo banco de dados de saúde nacional. P-valores bilaterais $<0,05$ foram considerados estatisticamente significativos.

Resultados: Durante um acompanhamento médio de 10 anos, foram registradas 71 mortes, das quais 62 foram devidas a DCV. Identificou-se um índice de mortalidade global e por DCV significativamente mais alto no grupo com nível de fetuína-A mais baixo que no grupo com nível de fetuína-A mais alto (44\% versus $24 \%, p=0,005 ; 48 \%$ versus $31 \%$, $p=$ 0,022 , respectivamente). Nas análises de risco proporcionais por regressão de Cox, detectou-se que a fetuína-A era um preditor independente de mortalidade global e por DCV.

Conclusões: A baixa concentração de fetuína-A está associada ao prognóstico de longo prazo ruim pós-STEMI, independentemente de fatores de risco cardiovascular tradicionais. Nossos achados fortaleceram estudos prévios demonstrando consistentemente o papel determinante dos mediadores anti-inflamatórios em síndromes coronárias agudas.

Palavras-chave: Infarto do Miocárdio com Supradesnível do Segmento ST; Doença da Artéria Coronariana; Mortalidade; Fetuína A; Globulinas; Anti-Inflamatórios; Ecocardiografia/métodos.

\footnotetext{
Abstract the prognosis of CAD patients is still controversial. (CVD) and all-cause mortality of ST-elevation acute myocardial infarction (STEMI). p-values $<0.05$ were considered statistically significant

Correspondência: Hakan Çakır •

Kartal Koşuyolu Training and Research Hospital - Cardiology - Kartal İstanbul, 34865 - Turquia

Artigo recebido em 10/10/2020, revisado em 14/01/2021, aceito em 24/02/2021

E-mail: dr.hcakir@gmail.com
}

Background: Fetuin-A is an anti-inflammatory and anti-calcification factor involved in the course of coronary artery disease (CAD). In line with these functions, fetuin-A has been investigated as a cardiovascular risk marker in many studies. However, the association between fetuin-A and

Objectives: The present study was conducted to identify the association between serum fetuin-A level and long-term cardiovascular disease

Methods: One hundred eigthy consecutive patients with STEMI were enrolled in the study. The study population was divided into subgroups (lower, $\leq 288 \mu \mathrm{g} / \mathrm{ml}$; and higher, $>288 \mu \mathrm{g} / \mathrm{ml}$ ) according to the median fetuin-A level. Clinical follow-up data was obtained by annual contact with the patients or family members by telephone. The causes of death were also confirmed by the national health database. Two-sided

Results: During a median follow-up of 10 years, 71 deaths were recorded, 62 of whom died from CVD. Both CVD and all-cause mortality were found to be significantly higher in the lower fetuin-A group than the higher fetuin-A group (44\% vs 24\%, $p=0.005 ; 48 \%$ vs 31\%, $p=0.022$, respectively). In Cox regression proportional hazard analyses, fetuin-A was found to be an independent predictor of CVD and all-cause mortality.

DOI: https://doi.org/10.36660/abc.20201057 
Conclusions: Low fetuin-A concentration is associated with a poor long-term prognosis after STEMI, regardless of the traditional cardiovascular risk factors. Our findings have strengthened previous studies that consistently demonstrate the determining role of anti-inflammatory mediators in acute coronary syndromes.

Keywords: ST Elevation Myocardial Infarction; Coronary Artery Disease; Mortality; Fetuin Alpha; Globulins; Anti- Inflammatory Agents; Echocardiography/methods.

\section{Introdução}

A fetuína-A, glicoproteína alfa-2-Heremans-Schmid, é uma proteína sérica abundantes que é produzida exclusivamente pelo fígado, pela língua, pela placenta e pelo tecido adiposo. ${ }^{1,2}$ Ela tem um papel crucial como inibidor fisiológico do receptor tirosina quinase associado à resistência a insulina e um reagente de fase aguda negativa. Ela também regula a remodelação óssea e o metabolismo do cálcio, sendo um inibidor importante da precipitação de sais de cálcio e calcificações vasculares. ${ }^{3}$

A inflamação é um processo-chave na aterosclerose. Existem evidências significativas do efeito prejudicial às citocinas próinflamatórias no sistema cardiovascular. ${ }^{4}$ Durante a inflamação, citocinas pró-inflamatórias, tais como IL-1ß e IL-6, reduzem a síntese da fetuína-A no fígado. Níveis de fetuína-A circulante reduzidos limitam as atividades de inúmeros mediadores antiinflamatórios, agravando, dessa forma, a resposta inflamatória. A fetuína-A é um dos inibidores de calcificação em tecido mole e na árvore vascular. ${ }^{5}$ A calcificação da artéria coronária é vista como índice da gravidade da doença vascular aterosclerótica e pode também prever eventos cardiovasculares adversos futuros.

Em alinhamento com essas funções, investigou-se a fetuína-A como marcador de risco cardiovascular em vários estudos. Estudos em populações com doença renal terminal (DRT) demonstram consistentemente que níveis de fetuína-A mais baixos são associados aos eventos de DCV e mortalidade global. ${ }^{6-8}$ Entretanto, a relação entre os níveis de fetuína-A sérica e o prognóstico de pacientes com doença arterial coronariana (DAC) ainda é controversa. Níveis altos de fetuína-A sérica estão associados a uma mortalidade de um ano mais baixa nos casos de síndromes coronárias agudas (SCA). ${ }^{9}$ Por outro lado, Parker et al., ${ }^{10}$ demonstraram que não se observou associação significativa entre a fetuína-A e evento de mortalidade ou DCV em pacientes com DAC estável. ${ }^{10}$ Weikert et al., ${ }^{11}$ relataram uma relação entre níveis de fetuína-A plasmática aumentada, e riscos mais altos de infarto do miocárdio (IM) e acidente vascular isquêmico em um estudo coorte de grande população. ${ }^{11}$

No presente estudo, o objetivo foi identificar a associação entre o nível de fetuína-A sérica e DCV de longo prazo e a mortalidade global de infarto do agudo do miocárdio por supradesnivelamento do segmento ST (STEMI). Levantou-se a hipótese de que níveis de fetuína-A mais baixos sejam um marcador prognóstico para mortalidade em longo prazo pósSTEMI, independentemente de outros fatores de risco de DCV.

\section{Métodos}

\section{População do estudo}

Entre maio e setembro de 2009, todos os pacientes (n: 195) diagnosticados com STEMI, e internados em até 12 horas após o surgimento dos sintomas, foram cadastrados inicialmente no estudo. Pacientes com choque cardiogênico em até 24 horas também foram incluídos. O STEMI foi diagnosticado na presença dos dois critérios abaixo: angina persistente por $\geq 20$ minutos e supradesnivelamento do segmento ST de $\geq 1 \mathrm{~mm}$ em $\geq 2$ derivações contíguas além de V2 ou V3, ou a presença de novos bloqueios de ramos do feixe esquerdo. Nas derivações V2 a V3, $2 \mathrm{~mm}$ de supradesnivelamento de ST nos homens, e 1,5 mm de supradesnivelamento de ST em mulheres, eram necessários para o diagnóstico de STEMI. Os pacientes foram manejados de acordo com as diretrizes, e o tratamento não foi afetado pela participação no cadastro. Preferiu-se a intervenção coronária percutânea (ICP) primária como técnica de reperfusão para toda a população do estudo. Os pacientes foram excluídos se alguma das características abaixo estivesse presente: lesão culpada ou crítica na artéria coronária principal esquerda, cirurgia de bypass da artéria coronária anterior, doença renal terminal (clearance de creatinina, $<15 \mathrm{~mL} / \mathrm{min}$ ), infecção ativa, doença inflamatória crônica, e malignidade conhecida. Ao final, a população do estudo consistiu em 180 pacientes. Foi realizado o exame físico completo e obteve-se um histórico médico detalhado de cada caso. Altura, peso e pressão arterial (PA) foram medidos por enfermeiros treinados, utilizando-se protocolos e procedimentos padrão. Hipertensão foi definida pela pressão arterial sistólica $\geq 140 \mathrm{mmHg}$, ou pressão arterial diastólica $\geq 90 \mathrm{mmHg}$, ou tratamento atual com qualquer medicamento hipertensivo. $\mathrm{O}$ índice de massa corporal (IMC) foi calculado como peso (kg)/ $\operatorname{altura}^{2}\left(\mathrm{~m}^{2}\right)$. O termo de consentimento informado foi obtido de todos os participantes. O estudo foi analisado e aprovado pelos comitês de ética institucionais.

\section{Medidas bioquímicas}

Foram coletadas amostras de sangue venoso dos participantes após um jejum noturno. O soro foi separado imediatamente das células por centrifugação a 3000 g por 10 minutos, armazenado a frio a $-70{ }^{\circ} \mathrm{C}$ até ser analisado. A fetuína-A sérica foi medida, utilizando-se um kit de ensaio de imunoabsorção enzimática de fetuína-A humana disponível comercialmente (BioVendor Laboratory Medicine, Inc., Brno, República Checa). Os coeficientes de variação intra e interensaio da fetuína-A foram menores que $8 \%$. Os níveis ultrassensíveis de PCR (PCR-us) foram medidos pelo método imunonefelométrico (Image Immunochemistry System; Beckman Coulter, Inc., Fullerton, CA, EUA). Outros parâmetros bioquímicos foram medidos utilizando-se métodos e kits comercialmente disponíveis.

\section{Ecocardiográfica}

Foi realizado um ecocardiograma bidimensional no hospital, antes da alta, para avaliar a fração de ejeção do ventrículo esquerdo (FEVE), utilizando-se a técnica de Simpson 
modificada. A análise foi realizada por dois observadores cegos em relação aos dados clínicos e angiográficos.

\section{Acompanhamento prospectivo e resultados}

Dados de acompanhamento clínico foram obtidos por contato telefônico anual com pacientes ou familiares após o ICP. As causas das mortes também foram confirmadas pelo banco de dados de saúde nacional. O acompanhamento foi concluído para todos os pacientes. Os resultados principais do estudo foram mortalidade cardiovascular (CID I00-199) e mortalidade global, que incluem mortalidade cardiovascular e morte devido a causas não cardíacas. Os pacientes passaram por triagens até o final de fevereiro de 2019 ou até a morte do paciente.

\section{Análise estatística}

Variáveis contínuas com distribuição normal são expressas como médias \pm desvio padrão (DP) e variáveis contínuas sem distribuição normal são expressas como mediana e faixa interquartil (FIQ). Dados categóricos são expressos como valores absolutos e porcentagens. O teste de KolmogorovSmirnov foi utilizado para testar a distribuição normal. As diferenças em variáveis contínuas entre os grupos foram analisadas utilizando o teste $t$ de amostras independentes ou o teste $U$ de Mann-Whitney, para variáveis com o sem distribuição normal, respectivamente. Variáveis categóricas e proporções foram analisadas pelo teste Qui-quadrado. A correlação entre a fetuína-A plasmática e os fatores de risco de DCV foi analisada utilizando-se a correlação de Pearson. Curvas de sobrevida para subgrupos de fetuína-A plasmática foram construídas pelo método Kaplan-Meier, e as diferenças em sobrevida foram avaliadas utilizando-se o teste de Log-rank.

A análise do modelo de risco proporcional de Cox foi usada para avaliar a associação entre a fetuína-A sérica e mortalidade global e por DCV no acompanhamento de 10 anos. O primeiro modelo era não padronizado. O modelo 2 foi padronizado para idade e sexo. Com base no modelo 2 , o modelo 3 foi padronizado além disso para fatores de risco cardiovascular tradicionais, tais como tabagismo, histórico de diabetes mellitus, histórico de hipertensão, e nível de colesterol total. Com base no modelo 3, o modelo 4 foi padronizado além disso para nível de creatinina e FEVE. A fetuína-A foi analisada separadamente como variável categórica e variável contínua. Os resultados dos modelos de regressão de Cox foram apresentados como razões de chance (RC) e intervalos de confiança (IC) de $95 \%$.

P-valores bilaterais $<0,05$ foram considerados estatisticamente significativos. As análises estatísticas foram realizadas com o software SPSS 20.0 (SPSS Inc., Chicago, IL, EUA).

\section{Resultados}

\section{Características clínicas e bioquímicas}

A população do estudo era composta de 180 pacientes com STEMI e dividida em subgrupos com fetuína-A mais baixa e mais alta, de acordo com a mediana do nível de fetuína-A na admissão. As características demográficas e bioquímicas dos níveis de fetuína-A na linha de base de acordo com os subgrupos (mais baixo, $\leq 288 \mu \mathrm{g} / \mathrm{ml}$; e mais alto, $>288 \mu \mathrm{g} / \mathrm{ml}$ ) estão resumidas na Tabela 1. Os pacientes do grupo com fetuína-A mais baixa eram mais velhos, e tinham uma prevalência mais alta de hipertensão e níveis mais altos de creatinina sérica e PCR-us em comparação ao grupo com fetuína-A mais alta. No ecocardiograma, os valores de FEVE eram mais baixos no grupo com fetuína-A mais baixa.

\section{Fetuína-A e fatores de risco de DCV}

A análise de correlação bivariada entre fetuína-A sérica e fatores de risco de DCV é apresentada na Tabela 2. O nível de fetuína-A estava inversamente correlacionado ao PCR-us, e diretamente correlacionado à glicemia de jejum após a padronização por sexo, idade e IMC.

\section{Resultados clínicos}

Os resultados clínicos são apresentados na Tabela 3. Durante um acompanhamento médio de 10 anos, foram registradas 71 mortes, das quais 62 foram devidas a DCV. Os índices de mortalidade global e por DCV foram significativamente mais altos no grupo com nível de fetuína-A mais baixo que no grupo com nível fetuína-A mais alto ( $44 \%$ versus $24 \%, p=0,005 ; 48 \%$ versus $31 \%, p=0,022$, respectivamente). As curvas de sobrevida acumulada demonstraram que os riscos de mortalidade global e por DCV aumentaram à medida que os níveis de fetuína-A diminuíram (Figura 1).

\section{Análise de regressão de Cox}

As RC para mortalidade cardiovascular e global associadas ao nível de fetuína-A plasmática são apresentadas na Tabela 4. Mesmo após a padronização para vários fatores confundidores (modelo 4), identificou-se que a fetuína-A é um preditor independente da mortalidade cardiovascular, como variável categórica e contínua $(R C=0,46$, IC 95\%: 0,27-0,78 e RC=0,997, IC 95\%: 0,994-0,999, respectivamente). Para a mortalidade global, após várias padronizações (modelo 4), identificou-se que a fetuína-A foi um preditor significativo como variável contínua ( $R C=0,997$, IC 95\%: 0,995-1,000). Ao mesmo tempo, houve uma tendência para a significância estatística da fetuína-A como variável categórica $(p=0,085)$.

\section{Discussão}

No presente estudo, demonstrou-se que os níveis mais altos de fetuína-A plasmática tinham risco mais baixo de mortalidade global e por DCV em pacientes com STEMI. Identificou-se que a associação era independente de fatores de risco estabelecidos para DCV, tais como, idade, tabagismo, hipertensão, diabetes mellitus, hiperlipidemia e creatinina. Além disso, identificou-se que o nível de fetuína-A plasmática estava inversamente correlacionado ao PCR-us, e diretamente correlacionado à glicemia de jejum. 
Tabela 1 - Características de linha de base da população do estudo estratificada pela mediana dos valores de fetuína-A na admissão $(\leq \mathbf{2 8 8} \mu \mathrm{g} / \mathrm{ml}$ versus $\leq \mathbf{2 8 8} \mu \mathrm{g} / \mathrm{ml}$ )

\begin{tabular}{|c|c|c|c|}
\hline \multirow[b]{2}{*}{ Variáveis } & \multicolumn{2}{|c|}{ Grupos de fetuína-A } & \multirow[b]{2}{*}{ p-valor } \\
\hline & $\begin{array}{c}\text { Fetuína-A mais baixa } \\
\begin{array}{c}(\leq \mathbf{2 8 8} \boldsymbol{\mu g} / \mathrm{ml}) \\
(\mathrm{n}: 90)\end{array}\end{array}$ & $\begin{array}{c}\text { Fetuína-A mais alta } \\
(\leq \mathbf{2 8 8} \mu \mathrm{g} / \mathrm{ml}) \\
(\mathrm{n}: 90)\end{array}$ & \\
\hline Idade, a & $60,5 \pm 9,9$ & $57,1 \pm 9,6$ & 0,019 \\
\hline Sexo, feminino/masculino & $12 / 78$ & $17 / 73$ & 0,311 \\
\hline Tabagismo, n (\%) & $59(65,6)$ & $51(56,7)$ & 0,221 \\
\hline Hipertensão, n (\%) & $32(35,6)$ & $19(21,1)$ & 0,032 \\
\hline Diabetes mellitus, n (\%) & $25(27,8)$ & $18(20,0)$ & 0,221 \\
\hline Colesterol total, mg/dl & $181 \pm 34$ & $182 \pm 34$ & 0,922 \\
\hline *Triglicérides, mg/dl & $141[102-194]$ & $137[103-181]$ & 0,595 \\
\hline LDL colesterol, mg/dl & $115 \pm 30$ & $113 \pm 31$ & 0,658 \\
\hline Hemoglobina, g/dl & $12,7 \pm 1,46$ & $13,0 \pm 1,18$ & 0,240 \\
\hline Creatinina, mg/dl & $1,10 \pm 0,74$ & $0,84 \pm 0,30$ & 0,002 \\
\hline PCR-us, mg/dl & $1,91 \pm 2,31$ & $0,86 \pm 1,56$ & $<0,001$ \\
\hline Pico de troponina I, ng/ml & $45,0 \pm 37,6$ & $35,1 \pm 34,1$ & 0,066 \\
\hline IM prévio, n (\%) & $6(6,6)$ & $4(5,5)$ & 0,756 \\
\hline IM da parede anterior, $\mathrm{n}(\%)$ & $35(38,9)$ & $28(31,1)$ & 0,274 \\
\hline IM inferior ou ventr. direito, n (\%) & $40(44,4)$ & $54(60,0)$ & 0,037 \\
\hline IM de parede posterolateral, n (\%) & $15(16,7)$ & $8(8,9)$ & 0,118 \\
\hline Tempo de reperfusão, $\mathrm{h}$ & $5,7 \pm 6,8$ & $6,4 \pm 10,0$ & 0,547 \\
\hline FEVE, \% & $42 \pm 7$ & $45 \pm 6$ & 0,016 \\
\hline
\end{tabular}

Os dados são expressos como média $\pm D P, n$ (\%) ou *mediana [FIQ]. IMC: índice de massa corporal; HDL: lipoproteína de alta densidade; PCR-us: proteína C reativa ultrassensivel; IM: infarto do miocárdio; LDL: lipoproteína de baixa densidade; FEVE: fração de ejeção ventricular esquerda.

Tabela 2 - Associação de fetuína-A plasmática com fatores de risco de doença cardiovascular

\begin{tabular}{|c|c|c|c|c|}
\hline \multirow[b]{3}{*}{ Variáveis } & \multicolumn{4}{|c|}{ Correlação com os níveis de fetuína-A plasmática } \\
\hline & \multicolumn{2}{|c|}{ Sem padronização } & \multicolumn{2}{|c|}{$\begin{array}{l}\text { Padronizado para } \\
\text { Sexo, idade e IMC }\end{array}$} \\
\hline & $\mathbf{r}$ & p-valor ${ }^{\dagger}$ & $\mathbf{R}$ & p-valor ${ }^{\ddagger}$ \\
\hline Idade, a & $-0,245$ & 0,001 & - & - \\
\hline $\mathrm{IMC}, \mathrm{kg} / \mathrm{m}^{2}$ & 0,031 & 0,681 & - & - \\
\hline PAS, $\mathrm{mm} \mathrm{Hg}$ & 0,084 & 0,263 & 0,038 & 0,616 \\
\hline $\mathrm{PAD}, \mathrm{mm} \mathrm{Hg}$ & 0,063 & 0,403 & 0,018 & 0,810 \\
\hline Glicemia jejum, mg/dl & 0,288 & $<0,001$ & 0,272 & $<0,001$ \\
\hline Colesterol total, $\mathrm{mg} / \mathrm{dl}$ & 0,056 & 0,456 & $-0,004$ & 0,961 \\
\hline Triglicérides, mg/dl & 0,083 & 0,268 & 0,073 & 0,333 \\
\hline $\mathrm{HDL}$ colesterol, mg/dl & 0,175 & 0,019 & 0,124 & 0,099 \\
\hline LDL colesterol, mg/dl & $-0,009$ & 0,903 & $-0,078$ & 0,302 \\
\hline PCR-us, mg/dl & $-0,276$ & $<0,001$ & $-0,233$ & 0,002 \\
\hline
\end{tabular}

IMC: indice de massa corporal; PAD: pressão arterial diastólica; HDL: lipoproteina de alta densidade; PCR-us: proteína C reativa ultrassensivel; LDL: lipoproteína de baixa densidade; PAS: pressão arterial sistólica. †Utilizada a correlação de Pearson. ‡Utilizada a análise de correlação parcial. 


\begin{tabular}{|c|c|c|c|}
\hline No acompanhamento de 10 anos & $\begin{array}{c}\text { Fetuína-A mais baixa } \\
(\leq \mathbf{2 8 8} \mu \mathrm{g} / \mathrm{ml}) \\
(\mathrm{n}: 90)\end{array}$ & $\begin{array}{c}\text { Fetuína-A mais alta } \\
(\leq \mathbf{2 8 8} \mu \mathrm{g} / \mathrm{ml}) \\
(\mathrm{n}: 90)\end{array}$ & p-valor \\
\hline Morte por DCV, n (\%) & $40(44)$ & $22(24)$ & 0,005 \\
\hline Mortalidade global, n, (\%) & $43(48)$ & $28(31)$ & 0,022 \\
\hline
\end{tabular}

DCV: doença cardiovascular.

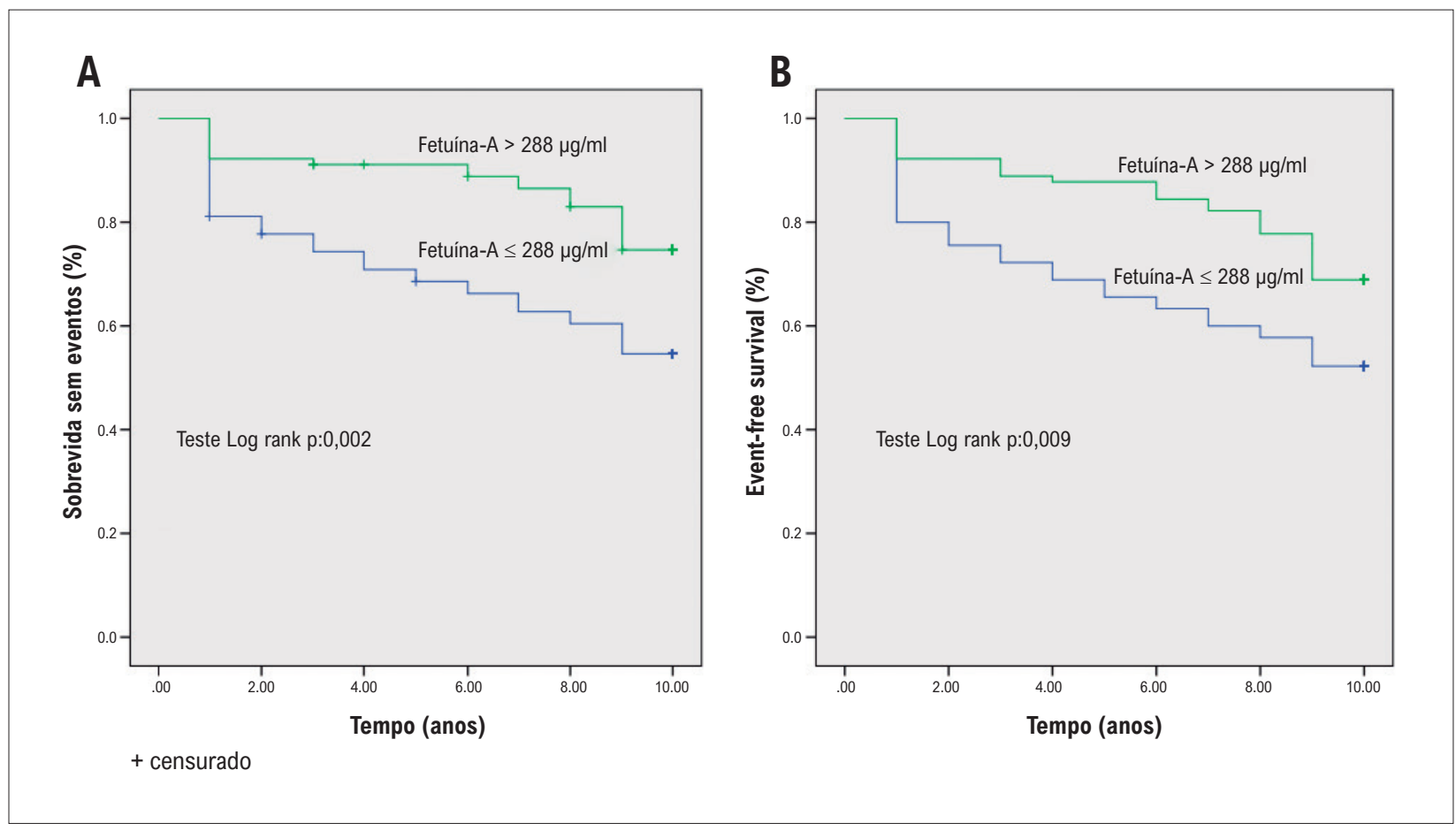

Figura 1 - Curvas de sobrevida de Kaplan-Meier de mortalidade por doença cardiovascular (A) e mortalidade global (B) de acordo com a fetuína-A.

Tabela 4 - Razões de risco baseadas nos modelos de regressão de Cox para estimar os efeitos dos níveis de fetuína-A na mortalidade global e por doença cardiovascular

\begin{tabular}{|c|c|c|c|c|}
\hline & \multicolumn{2}{|c|}{ Mortalidade por DCV } & \multicolumn{2}{|c|}{ Mortalidade global } \\
\hline & $\begin{array}{l}\text { Razão de risco } \\
\text { (IC 95\%) }\end{array}$ & p-valor & $\begin{array}{l}\text { Razão de risco } \\
\text { (IC 95\%) }\end{array}$ & p-valor \\
\hline \multicolumn{5}{|c|}{ Fetuína-A como variável categórica } \\
\hline Modelo 1 & $0,46(0.27-0.78)$ & 0,004 & $0,54(0.34-0.88)$ & 0,014 \\
\hline Modelo 2 & $0,46(0.27-0.79)$ & 0,005 & $0,55(0.33-0.89)$ & 0,016 \\
\hline Modelo 3 & $0,48(0.28-0.83)$ & 0,009 & $0,58(0.35-0,95)$ & 0,032 \\
\hline Modelo 4 & $0,55(0.31-0.96)$ & 0,036 & $0,64(0.38-1.06)$ & 0,085 \\
\hline \multicolumn{5}{|c|}{ Fetuína-A como variável contínua } \\
\hline Modelo 1 & 0,995 (0.993-0.998) & 0,001 & $0,996(0.993-0.998)$ & 0,001 \\
\hline Modelo 2 & $0,995(0.993-0.998)$ & 0,001 & $0,996(0.993-0.998)$ & 0,002 \\
\hline Modelo 3 & $0,996(0.993-0.999)$ & 0,003 & $0,996(0.994-0.999)$ & 0,005 \\
\hline Modelo 4 & 0,997 (0.994-0.999) & 0,038 & $0,997(0.995-1.000)$ & 0,034 \\
\hline
\end{tabular}

Modelo 1: Não padronizado. Modelo 2: padronizado para idade e sexo. Modelo 3: padronizado além disso para tabagismo, histórico de diabetes mellitus, histórico de hipertensão, e nivel de colesterol total. Modelo 4: padronizado além disso para nível de creatinina e fração de ejeção ventricular esquerda. IC: intervalo de confiança; DCV: doença cardiovascular. 
Estudos prévios investigando a relação entre a fetuína-A e a mortalidade global e cardiovascular tiveram resultados conflitantes. Concentrações mais baixas de fetuína-A previram o aumento da mortalidade global e por DCV nas populações com doença renal terminal, pacientes de diálise, e população em geral. ${ }^{7,12,13}$ Lim et al., ${ }^{14}$ estabeleceram que os índices de mortalidade de 6 meses eram significativamente mais altos em pacientes com STEMI com níveis baixos de fetuína-A em comparação aos pacientes com níveis altos de fetuína-A. ${ }^{14}$ Além disso, Chen et. al., ${ }^{15}$ demonstraram que níveis de fetuína-A plasmática mais baixos estavam associados com um aumento do risco de mortalidade global e por DCV em pacientes com DAC estável, independentemente de fatores de risco de DCV tradicionais. ${ }^{15}$ Contrariamente, Roos et al., ${ }^{16}$ demonstraram não existir associação significativa entre os níveis de fetuína-A sérica e eventos secundários de DCV em pacientes com DAC após o acompanhamento de 6 anos. ${ }^{16}$ Além disso, no estudo EPIC-Postdam, Weikert et al., ${ }^{17}$ demonstraram que pacientes com concentrações altas de fetuína-A tinham risco 4 vezes mais alto de infarto do miocárdio e acidente vascular isquêmico em comparação aos sujeitos com níveis baixos de fetuína-A. ${ }^{11}$

As razões subjacentes para tais discrepâncias mencionadas acima não foram claramente identificadas, mas algumas explicações foram propostas. A calcificação vascular e a inflamação podem ter um papel importante no desenvolvimento da aterosclerose e DCV. A fetuína-A pode ajudar a prevenir o desenvolvimento de doenças cardiovasculares por suas funções anti-inflamatórias e anticalcificação. ${ }^{17} \mathrm{~A}$ fetuína-A tem um papel crucial no caminho anti-inflamatório após uma isquemia miocárdica, provavelmente facilitando o início do processo de cura. ${ }^{4}$ Entretanto, a fetuína-A está envolvida na patogênese do diabetes mellitus tipo 2 ao inibir a fosforilação do receptor de insulina tirosina, o que leva à resistência a insulina. ${ }^{3}$ Uma meta-análise recente confirmou a associação, demonstrando que um incremento DP do nível de fetuína-A resulta em um risco $23 \%$ mais alto de diabetes mellitus tipo 2 incidente. ${ }^{18}$ Além disso, a fetuína-A poderia levar à ativação do receptor do tipo Toll 4 e à migração do macrófago, resultando em inflamação do tecido adiposo e disfunção do adipócito. ${ }^{19}$ Com base nesses fatos, o nível alto de fetuína-A, e não o nível baixo de fetuína-A, pode ser associado a aterosclerose e DCV. Esse efeito duplo da fetuína-A pode ter causado resultados heterogêneos em status e condições diferentes. Sugere-se que, nos estágios iniciais de DCV, a fetuína-A exacerba essa doença devido a seus efeitos de promoção da resistência à insulina e dislipidemia; entretanto, em estágios posteriores de DCV, altas concentrações de fetuína-A tiveram resultados positivos devido a seu efeito anti-inflamatório e capacidade de evitar o depósito de cálcio vascular. ${ }^{20,21}$ Outra explicação possível para esses resultados contraditórios é o fato de que a fetuína-A é uma proteína de fase aguda, que pode ser reduzida marcadamente no stress agudo. ${ }^{22}$ Embora essa condição reflita o equilíbrio da inflamação aguda em síndromes coronárias agudas, ela pode ter efeitos ilusórios em resultados de longo prazo. Por fim, nesses estudos, os níveis de fetuína-A foram medidas utilizando-se vários imunoensaios com sensibilidades e especificidades diferentes.
No estudo atual, identificou-se que a alta concentração de fetuína-A estava associada a um resultado favorável após o STEMI. Os efeitos inibidores da fetuína-A em processos inflamatórios podem explicar essa observação. A inflamação tem um papel crucial na patogênese da aterosclerose e da SCA. Após o SCA, a inflamação se espalha por todo o miocárdio e está envolvida na cicatrização e na recuperação das funções miocárdicas. ${ }^{23}$ Entretanto, o papel de mediadores anti-inflamatórios em contra-atacar e modular o processo inflamatório parece ser crítico para evitar a cicatrização inadequada. ${ }^{9}$ Nos casos em que a inflamação não pode ser suficientemente limitada por citocinas anti-inflamatórias, a inflamação pode levar a consequências desfavoráveis, tais como fibrose cardíaca, dilatação crônica, insuficiência cardíaca e arritmia. ${ }^{24}$ Há muitas evidências que demonstram os efeitos prejudiciais de mediadores pró-inflamatórios na SCA., ${ }^{4,25}$ Dados recentes também sugerem que marcadores anti-inflamatórios podem ter valor similar. ${ }^{26,27}$ Feistritzer et al., ${ }^{28}$ demonstraram que a fetuína-A baixa, associada a efeitos adversos de dimensões de enfartamento, função do ventrículo esquerdo, e remodelação após STEMI agudo. ${ }^{28}$ A baixa concentração de fetuína-A facilitará o processo inflamatório existente ${ }^{29}$ e a superprodução de citocinas cardiotóxicas, tais como o fator de necrose tumoral, ${ }^{30,31}$ que exporá os pacientes a um risco aumentado de remodelação de VE e recorrência de SCA. Além disso, a baixa concentração de fetuína-A pode ter um efeito deletério direto na função miocárdica. Merx et al., ${ }^{32}$ relataram recentemente o avanço da fibrose cardíaca, calcificação, função diastólica notadamente prejudicada, e tolerância a isquemia, bem como resistência a catecolamina nos corações de ratos knockout com fetuína-A. ${ }^{32}$

No cenário de STEMI, o efeito anti-inflamatório da fetuína-A pode ser mais dominante para se determinar o prognóstico que seus outros efeitos. Isso pode explicar a relação entre os níveis altos de fetuína-A e baixa mortalidade após o STEMI. Devido à associação bifásica da fetuína-A com doença cardiovascular, dependendo do estágio da aterosclerose, os valores de fetuína-A podem estar restritos a pacientes com STEMI para avaliar esse prognóstico.

\section{Limitações do estudo}

O presente estudo tem várias limitações a serem mencionadas. A limitação mais importante do presente estudo é que ele reflete uma experiência em um centro único com um número limitado de pacientes. Devido ao pequeno número de pacientes, o valor prognóstico da fetuína-A deve ser interpretado com cuidado nessa população. Nossos resultados são baseados em medições de fetuína-A de amostras de sangue únicas na admissão hospitalar. Os níveis de fetuína-A não foram avaliados após a fase aguda do infarto do miocárdio. A análise seria mais valiosa se a alteração da fetuína-A ao longo do tempo pudesse ser demonstrada com várias medidas. Por fim, este estudo não pôde considerar completamente todos os possíveis fatores confundidores associados aos níveis de fetuína-A plasmática ou desenvolvimento de DCV. 


\section{Conclusão}

O presente estudo demonstra que a baixa concentração de fetuína-A está associada a um prognóstico ruim para casos de STEMI no longo prazo. O impacto deletério de um processo inflamatório parece persistir após 10 anos de acompanhamento. Nossos achados fortaleceram estudos prévios demonstrando consistentemente o papel determinante dos mediadores antiinflamatórios em síndromes coronárias agudas. São necessários estudos randomizados de larga escala posteriores para explicar a utilidade clínica dos níveis de fetuína-A na previsão do prognóstico pós-STEMI no acompanhamento.

\section{Contribuição dos autores}

Concepção e desenho da pesquisa: Çakır Ha, Kanat S; Obtenção de dados: Kanat S, Çakır Hi; Análise e interpretação dos dados: Çakır Ha; Análise estatística: Çakır Ha, Çakır Hi;

\section{Referências}

1. Denecke B, Gräber S, Schäfer C, Heiss A, Wöltje M, Jahnen-Dechent W. Tissue distribution and activity testing suggest a similar but not identical function of fetuin-B and fetuin-A. Biochem J. 2003;376(Pt1):135-45.

2. Jialal I, Devaraj S, Bettaieb A, Haj F, Adams-Huet B. Increased adiose tissue secretion of fetuin-A, lipopolysaccharide-binding protein and highmobility group box protein 1 in metabolic syndrome. Atherosclerosis. 2015;241(1):130-7.

3. Xie WM, Ran LS, Jiang J, Chen YS, Ji HY, Quan XQ. Association between fetuin-A and prognosis of CAD: A systematic review and meta-analysis. Eur J Clin Invest. 2019;49(5):e13091.

4. Schernthaner C, Lichtenauer M, Wernly B, Paar V, Pistulli R, Rohm I, et al. Multibiomarker analysis in patients with acute myocardial infarction. Eur J Clin Invest. $2017 ; 47(9): 638-48$.

5. Wang H, Sama AE. Anti-inflammatory role of Fetuin-A in Injury and Infection. Curr Mol Med. 2012;12(5):625-33

6. Hermans MM, Brandenburg V, Ketteler M, Kooman JP, van der Sande FM, Boeschoten EW, et al. Association of serum fetuin-A levels with mortality in dialysis patients. Kidney Int. 2007;72(2):202-7.

7. Ketteler M, Bongartz P, Westenfeld R, Wildberger JE, Mahnken AH, Böhm $\mathrm{R}$, et al. Association of low fetuin-A (AHSG) concentrations in serum with cardiovascular mortality in patients on dialysis: a cross-sectional study. Lancet. 2003;361(9360):827-33.

8. Sági B, Peti A, Lakatos O, Gyimesi T, Sulyok E, Wittmann I, et al. Pro- and anti-inflammatory factors, vascular stiffness and outcomes in chronic hemodialysis patients. Physiol Int. 2020 Jul 2;107. Doi 10.1556/206000026 .

9. Lim P, Moutereau S, Simon T, Gallet R, Probst V, Ferrieres J, et al. Usefulness of fetuin-A and C-reactive protein concentrations for prediction of outcome in acute coronary syndromes (from the French Registry of Acute STElevation Non-ST-Elevation Myocardial Infarction [FAST-MI]. Am J Cardiol. 2013;111(1):31-7.

10. Parker BD, Schurgers LJ, Brandenburg VM, Christenson RH, Vermeer C, Ketteler M, et al. The associations of fibroblast growth factor 23 and uncarboxylated matrix Gla protein with mortality in coronary artery disease: the Heart and Soul Study. Ann Int Med. 2010;152(10):640-8.

11. Weikert C, Stefan N, Schulze MB, Pischon T, Berger K, Joost HG, et al. Plasma fetuin-A levels and the risk of myocardial infarction and ischemic stroke. Circulation. 2008;118(24):2555-62.
Redação do manuscrito: Çakır Ha, Tenekecioğlu E; Revisão crítica do manuscrito quanto ao conteúdo intelectual importante: Tenekecioğlu E.

\section{Potencial conflito de interesse}

Não há conflito com o presente artigo

\section{Fontes de financiamento}

O presente estudo não teve fontes de financiamento externas.

\section{Vinculação acadêmica}

Não há vinculação deste estudo a programas de pósgraduação.

12. Laughlin GA, Cummins KM, Wassel $\mathrm{CL}$, Daniels LB, Ix JH. The association of fetuin-A with cardiovascular disease mortality in older communitydwelling adults: the Rancho Bernardo study. J Am Coll Cardiol. 2012;59(19):1688-96.

13. Stenvinkel P, Wang K, Qureshi AR, Axelsson J, Pecoits-Filho R, Gao P, et al Low fetuin-A levels are associated with cardiovascular death: impact of variations in the gene encoding fetuin. Kidney Int. 2005;67(6):2383-92.

14. Lim P, Collet JP, Moutereau S, Guigui N, Mitchell-Heggs L, Loric S, et al. Fetuin- $\mathrm{A}$ is an independent predictor of death after ST-elevation myocardial infarction. Clin Chem. 2007;53(10):1835-40.

15. Chen X, Zhang Y, Chen Q, Li Q, Li Y, Ling W. Lower plasma fetuin-A levels are associated with a higher mortality risk in patients with coronary artery disease. Arterioscler Thromb Vasc Biol. 2017;37(11):2213-9

16. Roos M, von Eynatten M, Heemann U, Rothenbacher D, Brenner $\mathrm{H}$, Breitling LP. Serum fetuin-A, cardiovascular risk factors, and sixyear follow-up outcome in patients with coronary heart disease. Am J Cardiol. 2010;105(12):1666-72.

17. Mori K, Emoto M, Inaba M. Fetuin-A: a multifunctional protein. Recent Pat Endocr Metab Immune Drug Discov. 2011;5(2):124-46.

18. Guo VY, Cao B, Cai C, Cheng KK, Cheung BMV. Fetuin-A levels and risk of type 2 diabetes mellitus: a systematic review and meta-analysis, Acta Diabetol. 2018;55(1):87-98.

19. Pal D, Dasgupta S, Kundu R, Maitra S, Das G, Mukhopadhyay S, et al. Fetuin-A acts as an endogenous ligand of TLR4 to promote lipid-induced insulin resistance. Nature Med. 2012;18(8):1279-85.

20. Jirak P, Stechemesser L, Moré E, Franzen M, Topf A, Mirna M, et al. Clinica implications of fetuin-A. Adv Clin Chem. 2019;89:79-130.

21. Zhao ZW, Lin CG, Wu LZ, Luo YK, Fan L, Dong XF etal. Serum fetuin-A levels are associated with the presence and severity of coronary artery disease in patients with type 2 diabetes. Biomarkers. 2013;18(2):160-4

22. Basar N, Sen N, Kanat S, Ozlu MF, Ozcan F, Cay S, et al. Lower fetuin-A predicts angiographic impaired reperfusion and mortality in ST-elevation myocardial infarction. J Investig Med. 2011;59(5)_:816-22.

23. Abbate A, Bonanno E, Mauriello A, Bussani R, Biondi-Zoccai GG, Liuzzo $\mathrm{G}$, et al. Widespread myocardial inflammation and infarct-related artery patency. Circulation. 2004; 110(1):46-50.

24. Nian M, Lee P, Khaper N, Liu P. Inflammatory cytokines and postmyocardia infarction remodeling. Circ Res. 2004;94(12):1543-53. 


\section{Artigo Original}

25. de Winter RJ, Bholasingh R, Lijmer JG, Koster RW, Gorgels JP, Schouten Y, et al. Independent prognostic value of $\mathrm{C}$-reactive protein and troponin I in patients with unstable angina or non-Q-wave myocardial infarction. Cardiovasc Res. 1999;42(1):240-5.

26. Ong SB, Hernández-Reséndiz S, Crespo-Avilan GE, Mukhametshina RT, Kwek XY, Cabrera-Fuentes HA, et al. Inflammation following acute myocardial infarction: Multiple players, dynamic roles, and novel therapeutic opportunities. Pharmacol Ther. 2018 Jun; 186: 73-87.

27. Tziakas DN, Chalikias GK, Kaski JC, Kekes A, Hatzinikolaou EI, Stakos DA, et al. Inflammatory and anti-inflammatory variable clusters and risk prediction in acute coronary syndrome patients: a factor analysis approach. Atherosclerosis. 2007;193(1):196-203.

28. Feistritzer HJ, Klug G, Reinstadler SJ, Gröber MT, Mair J, Kirchmair R, et al. Fetuin-A is related to infarct size, left ventricular function and remodelling after acute STEMI. Open Heart. 2015 Jun 26; 2(1): e000244.
29. Ombrellino $M$, Wang $H$, Yang $H$, Zhang $M$, Vishnubhakat J, Frazier A, et al. Fetuin, a negative acute phase protein, attenuates TNF synthesis and the innate inflammatory response to carrageenan. Shock. 2001;15(3):181-5.

30. Kelly RA, Smith TW. Cytokines and cardiac contractile function. Circulation. 1997; 95(4): 778-81.

31. Krown KA, Yasui K, Brooker MJ, Dubin AE, Nguyen C, Harris GL, et al. TNF alpha receptor expression in rat cardiac myocytes: TNF alpha inhibition of L-type Ca2 + current and Ca2 + transients. FEBS Lett.1995;(1-2):24-30.

32. Merx MW, Schäfer C, Westenfeld R, Brandenburg V, Hidajat S, Weber C, et al. Myocardial stiffness, cardiac remodeling, and diastolic dysfunction in calcification-prone fetuin-A-deficient mice. J Am Soc Nephrol.2005;16(11):3357-64. 